\title{
INEQUALITIES IN HILBERT MODULES OF MATRIX-VALUED FUNCTIONS
}

\author{
ADHEMAR BULTHEEL
}

\begin{abstract}
The classical Cauchy-Schwarz inequality and extremality properties of reproducing kernels are generalized for a module of matrix-valued functions on which a matrix-valued inner product is defined. Reference to an application in the field of linear prediction of multivariate stochastic processes is made.
\end{abstract}

$\mathbf{F}$ denotes the field of real numbers $\mathbf{R}$ or the field of complex numbers $\mathbf{C}$. $K=$ $\mathbf{F}^{n \times n}$ is the ring of $n \times n$ matrices with their entries in $\mathbf{F}$. We assume

(1) $M$ is a (left) $K$-module of $K$-valued functions on a set $\Omega$, and

(2) $\langle\cdot, \cdot\rangle$ is a $K$-valued nonnegative hermitian sesquilinear function on $M \times M$, i.e. for $F, G, H \in M$ and $A, B \in K$,

(P1) $\langle F, F\rangle \geq 0$,

(P2) $\langle F, G\rangle=\langle G, F\rangle^{*}$,

(P3) $\langle A F+B G, H\rangle=A\langle F, H\rangle+B\langle G, H\rangle$

(* denotes the adjoint of a matrix).

$M$ with $\langle\cdot, \cdot\rangle$ is called a Hilbert module if $\langle F, F\rangle=0$ iff $F=0$. Note that $\langle\langle\cdot, \cdot\rangle\rangle=\operatorname{trace}\langle\cdot, \cdot\rangle$ is an $\mathbf{F}$-valued nonnegative hermitian sesquilinear functional when $M$ is taken over the subring of matrices of the form $a I, a \in \mathbf{F}$. Thus the classical Cauchy-Schwarz inequality holds, i.e.

$$
|\langle\langle F, G\rangle\rangle| \leq\langle\langle F, F\rangle\rangle^{1 / 2}\langle\langle G, G\rangle\rangle^{1 / 2} \quad \forall F, G \in M .
$$

Note further that $\langle F, F\rangle=0 \Leftrightarrow\langle\langle F, F\rangle\rangle=0$. Define the equivalence relation $\sim$ on $M$ by $F \sim G$ iff $\langle F-G, F-G\rangle=0$, and define for each $F \in M$ the equivalence class $[F]_{e}=\{G \mid G \sim F\}$. Then $[F]_{e}=F+[0]_{e}=\{F+G \mid G \sim 0\}$.

The following result is the $K$-valued generalization of the Cauchy-Schwarz inequality.

THEOREM 1. For $M,\langle\cdot, \cdot\rangle$ as in (1), (2) we have

$$
\langle F, G\rangle\langle G, G\rangle^{+}\langle G, F\rangle \leq\langle F, F\rangle \quad \forall F, G \in M .
$$

(If $A$ is hermitian, $A^{+}$denotes any hermitian $\{2\}$-inverse of $A$, i.e. [2] $A^{+}$is hermitian and solves $A^{+} A A^{+}=A^{+}$. In particular we could take for $A^{+}$the unique Moore-Penrose generalized inverse.)

ProOF. Properties (P1)-(P3) imply that

$0 \leq\langle A F+B G, A F+B G\rangle=A\langle F, F\rangle A^{*}+B\langle G, G\rangle B^{*}-A\langle F, G\rangle B^{*}-B\langle G, F\rangle A^{*}$.

Replace $A$ by $I$ and $B$ by $\langle F, G\rangle\langle G, G\rangle^{+}$; then the result follows.

Received by the editors July 21, 1981 and, in revised form, October 12, 1981.

1980 Mathematics Subject Classification. Primary 46C10, 47D15.

Key words and phrases. Cauchy-Schwarz, reproducing kernel, matrix optimization.

(C) 1982 American Mathematical Society 0002-9939/81/0000-0289/\$02.25 
As in the classical case of semidefinite inner product spaces, one can show with the aid of Theorem 1 that

$$
[0]_{e}=M \cap M^{\perp}=M \cap\{F \in M \mid\langle F, G\rangle=0 \forall G \in M\}
$$

[3], i.e. the neutral and isotropic elements of $M$ coincide. We have for $F_{1} \sim F_{2}$ and $G_{1} \sim G_{2}$ that $\left\langle F_{1}, G_{1}\right\rangle=\left\langle F_{2}, G_{2}\right\rangle .\left\langle[F]_{e},[G]_{e}\right\rangle_{e}=\langle F, G\rangle$ is thus well defined and $M_{e}=\left\{[F]_{e} \mid F \in M\right\}$ is a Hilbert module under $\langle\cdot, \cdot\rangle_{e}$.

Let $M,\langle\cdot, \cdot\rangle$ be as in (1), (2). A $\langle\cdot, \cdot\rangle$-compatible reproducing kernel is a function $k(\cdot, \cdot): \Omega \times \Omega \rightarrow K$ such that

(K1) $k(\cdot, \omega) \in M \forall \omega \in \Omega$, and

(K2) $\forall F \in M: \exists G \in[F]_{e}$ such that $\langle F, k(\cdot, \omega)\rangle=G(\omega) \forall \omega \in \Omega$.

Note when $[0]_{e}=\{0\}$, i.e. $\langle F, F\rangle=0 \Leftrightarrow F=0$; then $k(\cdot, \cdot)$ is the usual Aronszajn type $(K$-valued) reproducing kernel $[\mathbf{1}]$.

We now show that $k(\cdot, \cdot)$ solves some optimization problems as in the classical case [8, Theorem III.3, p. 44].

THEOREM 2. Let $M,\langle\cdot, \cdot\rangle, k(\cdot, \cdot)$ be as in (1), (2), (3). Let $\alpha \in \Omega$ be fixed and let $k(\alpha, \alpha)$ be invertible. Then (with respect to the $\leq$-ordering of matrices)

(a) $\min \{\langle F, F\rangle \mid F(\alpha)=I, F \in M\}=k(\alpha, \alpha)^{-1}$, where the minimum occurs only for the elements $F \in\left[k(\alpha, \alpha)^{-1} k(\cdot, \alpha)\right]_{e}$ that satisfy $F(\alpha)=I$ (so the minimizing value is unique when $\left.[0]_{e}=\{0\}\right)$;

(b) when $[0]_{e}=\{0\}, \max \left\{F(\alpha)^{*} F(\alpha) \mid\langle F, F\rangle=I, F \in M\right\}=k(\alpha, \alpha)$, where the maximum occurs only for $F$ of the form $F(\cdot)=A k(\cdot, \alpha)$ where $A$ is any matrix such that $A^{*} A=k(\alpha, \alpha)^{-1}$ (for example we may take $\left.A=\left(k(\alpha, \alpha)^{-1}\right)^{1 / 2}\right)$.

Proof. (a) For any $F \in M$ with $F(\alpha)=I$, Theorem 1 gives

$$
\langle F, F\rangle \geq\langle F, k(\cdot, \alpha)\rangle\langle k(\cdot, \alpha), k(\cdot, \alpha)\rangle^{-1}\langle k(\cdot, \alpha), F\rangle=k(\alpha, \alpha)^{-1} .
$$

This lower bound for $\langle F, F\rangle$ is obtained by $F=S \triangleq k(\alpha, \alpha)^{-1} k(\cdot, \alpha)$ as one can easily verify. This proves that $k(\alpha, \alpha)^{-1} k(\cdot, \alpha)$ is a solution. Any other solution $G$ has to satisfy $G(\alpha)=I$ and $\langle G, G\rangle=k(\alpha, \alpha)^{-1}$. The expansion of $\langle S-G, S-G\rangle$ shows that it equals zero, thus $G \in[S]_{e}$ and $G(\alpha)=I$.

(b) If we take in Theorem $1 k(\cdot, \alpha)$ for $F$ and if $G$ satisfies $\langle G, G\rangle=I$, we have

$$
k(\alpha, \alpha)=\langle k(\cdot, \alpha), k(\cdot, \alpha)\rangle \geq\langle k(\cdot, \alpha), G\rangle\langle G, G\rangle^{+}\langle G, k(\cdot, \alpha)\rangle=G(\alpha)^{*} G(\alpha) .
$$

The upper bound $k(\alpha, \alpha)$ for $G(\alpha)^{*} G(\alpha)$ is obtained for $G(\cdot)=A k(\cdot, \alpha)$ with $A$ as above. Clearly $\langle G, G\rangle=I$ and $G(\alpha)^{*} G(\alpha)=k(\alpha, \alpha)$. If $F$ is any other solution of this problem, then it must also satisfy $F(\alpha)^{*} F(\alpha)=k(\alpha, \alpha)$ and $\langle F, F\rangle=I$. We claim that then $F$ has the form $F(\cdot)=\left(F(\alpha)^{*}\right)^{-1} k(\cdot, \alpha)$ and thus is of the form required by the theorem. That $F$ indeed has this form can be seen by checking that

$$
\left\langle F(\cdot)-\left(F(\alpha)^{*}\right)^{-1} k(\cdot, \alpha), F(\cdot)-\left(F(\alpha)^{*}\right)^{-1} k(\cdot, \alpha)\right\rangle=0
$$

and thus $F(\cdot)=\left(F(\alpha)^{*}\right)^{-1} k(\cdot, \alpha)$ because $[0]_{e}=\{0\}$.

THEOREM 3. Let $M,\langle\cdot, \cdot\rangle$ be as in (1), (2) and let $M_{e}$ be the Hilbert module as defined before. Let $\left\{\Phi_{k}\right\}_{1}^{N}$ be such that $\left\{\left[\Phi_{k}\right]_{e}\right\}_{1}^{N}$ span $M_{e}$ over $K$ and for $1 \leq j$, $k \leq N,\left\langle\Phi_{j}, \Phi_{k}\right\rangle=\delta_{j k} I$. Then $k\left(\omega_{1}, \omega_{2}\right)=\sum_{i=1}^{N} \Phi_{i}\left(\omega_{2}\right) \Phi_{i}\left(\omega_{1}\right)$ is a $\langle\cdot, \cdot\rangle$-compatible reproducing kernel for $M$. 
ProOF. Write $F \in M$ as $F=\sum_{k=1}^{N} A_{k} \Phi_{k}+\Phi$ with $\Phi \in[0]_{e}$. Then verify that $\langle F(\cdot), k(\cdot, \omega)\rangle=\sum_{k=1}^{N} A_{k} \Phi_{k} \in[F]_{e}$ and the theorem is proved.

Application of Theorem 2(a). Consider the $K$-module $M$ of matrix-valued functions analytic inside the unit disk of the complex plane and square integrable over the unit circle, i.e. for which we can define $\forall F, G \in M$,

$$
\langle F, G\rangle=\int_{[-\pi, \pi)} F\left(e^{i \theta}\right) H(d \theta) G\left(e^{i \theta}\right)^{*}
$$

in the sense of [9]. $H(\cdot)$ is an $n \times n$, nontrivial, nonnegative, hermitian matrixvalued measure on the Borel sets of the real interval $[-\pi, \pi)$. Of course we have a Hilbert module $M_{e}$ of equivalence classes as introduced before. $F \sim G$ iff $F=G$ a.e. with respect to $H(\cdot)$ as in [9]. Consider now the class of functions $M_{N}$ spanned by $\left\{\left[B_{k}\right]_{e}\right\}_{0}^{N}$ over $K$ such that $\left(M_{N}\right)_{e}$ is a submodule of $M_{e}$. Let $k_{N}(\cdot, \cdot)$ be a $\langle\cdot, \cdot\rangle$-compatible reproducing kernel for $M_{N}$. In $M_{N}$ we minimize $\langle F, F\rangle$ with $F$ constrained by the condition $F(\alpha)=0$ for some $\alpha,|\alpha|<1$. By Theorem 2(a), this optimum is $k_{N}(\alpha, \alpha)^{-1}$, and this is obtained by any function $F$ of the class $\left[k_{N}(\alpha, \alpha)^{-1} k_{N}(\cdot, \alpha)\right]_{e}$ for which $F(\alpha)=I$.

We consider two important examples.

(a) Take $B_{k}=z^{k} I, k=0,1, \ldots, N . M_{N}$ consists of matrix polynomials of degree at most $N$. The solution of the previously described optimization problem is given by Szegö's orthogonal polynomials [5] and has applications in AR filtering [10].

(b) Take $B_{0}=I, B_{k}=\left(\left(z-\alpha_{k}\right) /\left(1-\bar{\alpha}_{k} z\right)\right) B_{k-1},\left|\alpha_{k}\right|<1, k=1,2, \ldots, N$. $M_{N}$ consists of rational matrix functions. The optimization problem is now related to the ARMA filtering problem [7]. Recursive construction of the reproducing kernels for increasing $N$ is possible by the Nevanlinna-Pick algorithm [6, 4]. It is a nice generalization of the Schur-Szegö recursion in the polynomial case.

One last remark: The solution given is possible only if $k_{N}(\alpha, \alpha)$ is invertible. In both previous examples $B_{0}$ is constant, and thus $k_{0}(\alpha, \alpha)=\Phi_{0} \Phi_{0}^{*}$ where $\Phi_{0} \in K$ is a normalized version of $B_{0}$, e.g. $\Phi_{0}=\left(\int_{[-\pi, \pi)} H(d \theta)\right)^{-1 / 2}>0$ if $H$ is nontrivial. If $\left\{\Phi_{k}\right\}_{0}^{N}$ is an orthonormal generating set dervied from $\left\{B_{k}\right\}_{0}^{N}$ with the properties of Theorem 3, then

$$
k_{j}(\alpha, \alpha)=\sum_{i=1}^{j} \Phi_{i}(\alpha) \Phi_{i}(\alpha)^{*} \geq k_{0}(\alpha, \alpha)>0,
$$

and thus $k_{j}(\alpha, \alpha)$ is nonsingular for $j>0$.

\section{REFERENCES}

1. N. Aronzajn, Theory of reproducing kernels, Trans. Amer. Math. Soc. 68 (1950), 337-404.

2. A. Ben-Israel and T. N. E. Greville, Generalized inverses, theory and applications, Wiley, New York, 1974.

3. J. Bognar, Indefinite inner product spaces, Springer-Verlag, Berlin, 1974.

4. A. Bultheel, Orthogonal matrix functions related to the multivariable Nevanlinna-Pick problem, Bull. Soc. Math. Belg. Ser. B 31 (1980), 149-170.

5. P. Delsarte, Y. Genin and Y. Kamp, Orthogonal polynomial matrices on the unit circle, IEEE Trans. Circuits and Systems CAS-25 (1978), 1051-1059.

6. - The Nevanlinna-Pick problem for matrix valued functions, SLAM J. Appl. Math. 36 (1978), 47-61. 
7. P. Dewilde and H. Dym, Lossless chain scattering matrices and optimum linear prediction: the vector case, Circuit Theory 9 (1981), 135-175.

8. H. Meschkowski, Hilbertsche Räume mit Kernfunktionen, Springer-Verlag, Berlin, 1962.

9. M. Rosenberg, The square integrability of matrix valued functions with respect to a non-negative hermitian measure, Duke J. Math. 31 (1964), 291-298.

10. N. Wiener and P. Masani, The prediction theory of multivariable stochastic processes. I: Regularity conditions, Acta Math. 98 (1957), pp. 111-150; II: The linear predictor, Acta Math. 99 (1958), 93-139.

DePARTMENT OF COMPUTER SCIENCE, KATHOLIEKE UNIVERSiteit LeuVEN, CelestijnenlaAn 200A, B-3030 Heverlee, Belgium 